\title{
TRANSCRANIAL DIRECT CURRENT STIMULATION (tDCS) IN PREGNANT WOMEN WITH DEPRESSIVE SYMPTOMS
}

\author{
Lõokene M, The North Estonia Medical Centre
}

Background: Major depressive disorder (MDD) is the most common morbidity in pregnancy, affecting approximately $10 \%$ of pregnant women (Gavin et al., 2005). Untreated or incompletely treated depression can have profound adverse implications for maternal and fetal health. It has been estimated that approximately $80 \%$ of pregnant women with depression do not receive adequate treatment (Sreeraj et al., 2016). As a noninvasive method lacking systemic effects, transcranial direct current stimulation (tDCS) provides a promising alternative strategy for the treatment of depression and other mental disorders during pregnancy. However, only few case reports are currently available describing efficacy and safety of tDCS in treatment of prenatal depression.

Objectives: The aim of this study was to assess the effectiveness and tolerability of tDCS in pregnant women with depressive symptoms.

Materials and Methods: Seven pregnant women with the diagnosis of major depressive disorder or anxiety disorder received tDCS and a clinical evaluation with the 24-item Hamilton Rating Scale for Depression at baseline and posttreatment. Adverse events were collected using a questionnaire. tDCS was delivered using Sooma tDCS ${ }^{\mathrm{TM}}$ portable device. The anode was placed on the scalp over the left DLPFC (F3) and the cathode on the right DLPFC (F4). A direct current of $2 \mathrm{~mA}$ was delivered for 30 minutes 5 times per week for 2-3 weeks, further maintenance treatment was administered according to individual needs.

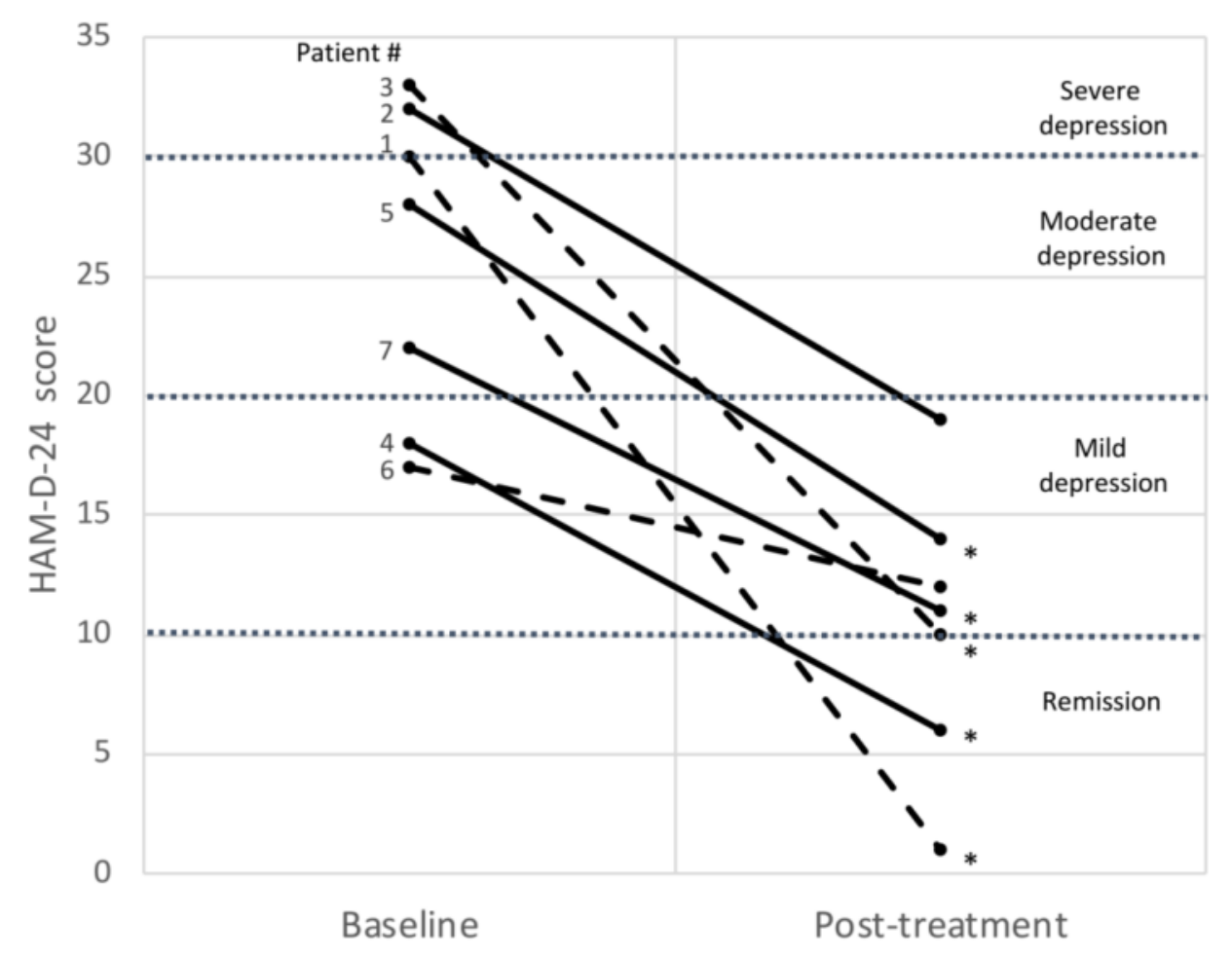

Results: Total depression scores decreased in all participants after the tDCS treatment, two patients achieved clinical remission. No serious adverse effects were reported.

Conclusions: These results suggest that tDCS is a welltolerated and effective non-pharmacological treatment option for prenatal depression.

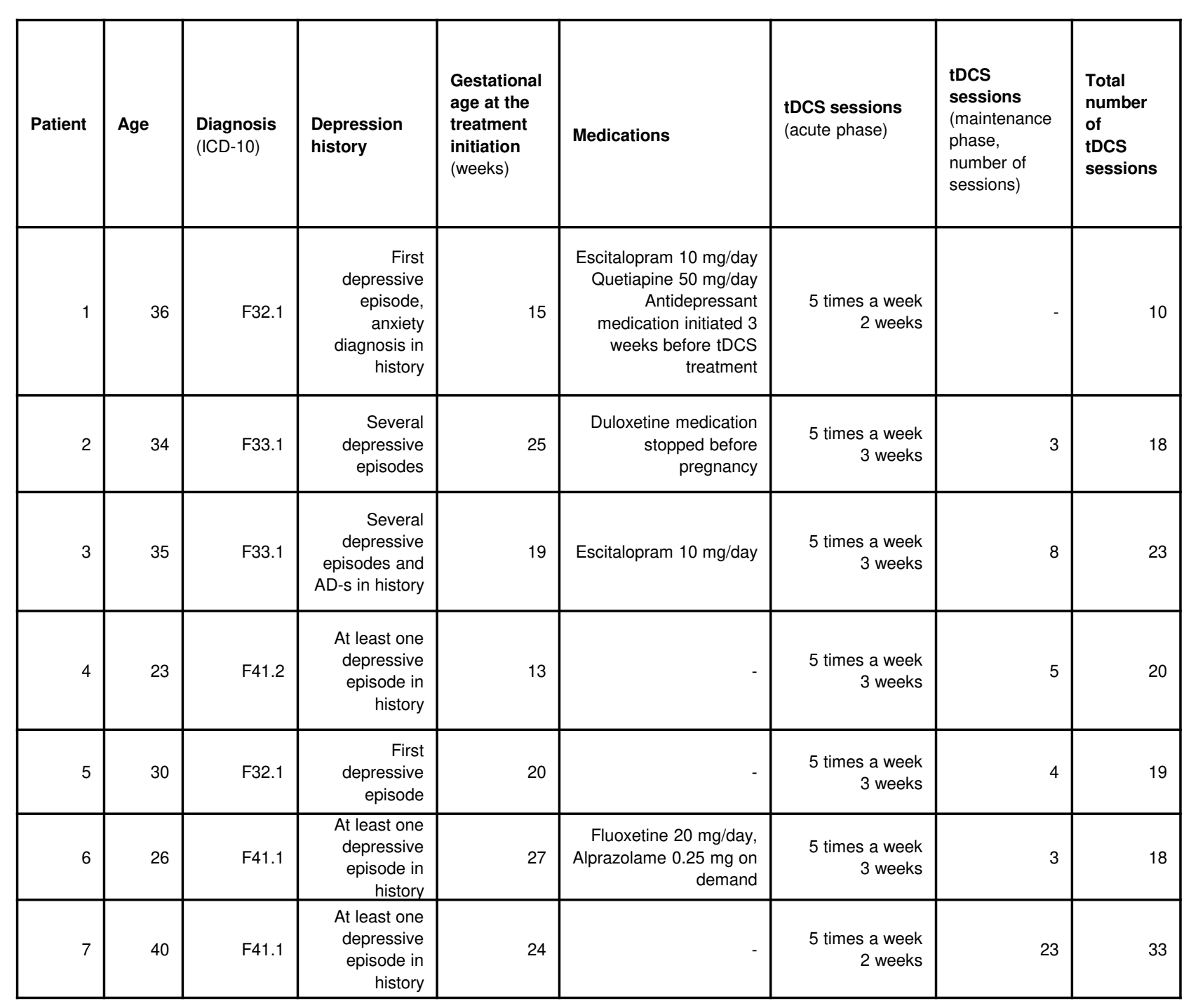

\section{Sources:}

- Data supporting the findings are available in The North Estonia Medical Centre electronic health records

- Gavin, N. I., Gaynes, B. N., Lohr, K. N., Meltzer-Brody, S., Gartlehner, G., \& Swinson, T. (2005). Perinatal depression: A systematic review of prevalence and incidence. Obstet Gynecol. 2005 Nov;106(5 Pt 1):1071-83

- Sreeraj, V. S., Bose, A., Shanbhag, V., Narayanaswamy, J. C., Venkatasubramanian, G., \& Benegal, V. (2016). Monotherapy with tDCS for Treatment of Depressive Episode during Pregnancy: A Case Report. Brain Stimul. 2016 MayJun;9(3):457-58 